\title{
FPGA Implementation of a Novel Oversampling DeadBeat Controller for PMSM Drives
}

\begin{abstract}
This paper presents a novel Oversampling DeadBeat (OS-DB) current control approach for Permanent Magnet Synchronous Motors (PMSMs) drives capable of operating at a controller sampling frequency multiple of the power converter switching frequency. Model based controllers suffer form heavy computational demand and performance degradation due to parameters uncertainties. The proposed controller concurrently with FPGA implementation permits to achieve a constant switching frequency and an optimal current ripple along with a high current loop bandwidth and robust behaviour to parameters variation. A disturbance observer has been added to the proposed controller in order to compensate for the converter voltage distortions. The proposed control strategy is tested through both simulations and experiments.
\end{abstract}

Index Terms-DeadBeat Control, Model Predictive Control, Oversampling, Permanent Magnet Synchronous Motors.

\section{INTRODUCTION}

In the field of variable-speed AC drives PMSMs, compared to other motor types like induction motors, offer several advantages. They have higher efficiency, higher performance, compact construction and higher torque per volume ratio. High performance electrical drives require efficient inner current control loops due to the intrinsic relationship between current quality response and torque control. Among the controllers which can achieve high current loop bandwidth there are Predictive Controllers [1]. Of particular interest because of its dynamic performances is the DeadBeat (DB) control which is able to force the control error to zero in a short finite time resulting in a fast transient response. Compared to other predictive control approaches like Finite Control Set Model Predictive Controller (FCS-MPC), DB combines good dynamic performance with a constant switching frequency. FCSMPC in fact, do not utilise a modulator leading to variable switching frequency and, often, to a suboptimal current ripple [2], [3]. Because of these reasons the DB controller has been chosen over other controllers in many applications such as uninterruptible power supply (UPS) [4], [5], PWM rectifiers, active filters control [6] and motor drive control [7]-[10]. When the MPCs are implemented in a full digital system it is well-known that one of their main limitations is their complex implementation [1] which often leads to an inevitable delay between measurements and control action to the inverter. For the DB controller this results in doubling the time necessary to bring to zero the current error. In one controller sample time it is necessary to measure, calculate and actuate the control actions, which is often not possible for standard DSP digital controllers, leading to the introduction of the sample time delay compensation. However, increasing the processing speed by using specific digital hardware technology such as field programmable gate arrays (FPGA) allows to significantly reduce the calculation time and enabling to measure, compute and actuate in a single controller sample time. The advantages of FPGA-based MPC have been investigated in [11]-[14]. The implementation of the standard DB controller on a FPGA controller can be found in [15], [16]. The other main limitation of predictive controllers as well as DB controllers is the strong dependency of their performances from model parameters. Machine parameters deviations from nominal ones or simply lack of knowledge of their values, results in a significant reduction of both transient and steady state performances as reported in [2], [6], [8], [17]-[19]. The DB controller performances are deteriorated not only by parameters uncertainties but also from the converter voltage non-linearities such as the dead times (DT), voltage drops and unideal turn-on and turn-off of the devices. Previous work has been done combining the DB controller with a standard PI to reduce the performance deterioration due to voltage non-linearities and parameters mismatches [7], while in [5] the control sensitivity from model uncertainties is reduced by introducing a disturbance observer for the DB controller.

This paper investigates a new DB approach in order to operate at an increased controller sampling frequency without increasing the commutation frequency of the power converter. The proposed OS-DB controller is obtained by discretizing the system equations over a variable sample time $T_{s}(i)$ resulting in an increased control sampling frequency. Although the proposed oversampling approach increase the robustness to machine parameters variation, on the other hand it introduces a distortion in the control voltages fed into the PWM. To eliminate this behaviour a compensation strategy has been developed and successfully validated. Furthermore, a reduced order Luenberger disturbance observer has been added to OSDB controller to remove steady-state errors due to inverter non-linearities and parameters mismatch. The whole controller has been implemented in FPGA in order to increase the transient performances and avoid the step delay compensation. The proposed technique permits to achieve a constant switching frequency and an optimal current ripple along with a high current loop bandwidth and robust behaviour to parameter variation. The paper is organized as follows: in Section II PMSM model is presented. Section III presents the standard DBCC for a PMSM. Sections IV and IV-A illustrate the new variable sample time OS-DB while the disturbance observer is presented in Section V. Simulation and experimetal results are shown in Section VI and VII respectively. Finally, Section VIII reports the conclusions.

\section{PMSM Mathematical Model}

The considered electrical system is a 3-phase, p-pole, wyeconnected permanent magnet synchronous machine [20]. The 
stator windings are identical sinusoidally distributed windings, displaced of $120^{\circ}$ with resistance $R_{s}$. The magnetic axes of the stator windings are denoted by the $a s, b s$ and $c s$ axes. The machine voltage and flux equations in the abc reference frame are reported in matrix form in (1)

$$
\left\{\begin{array}{l}
V_{a b c}=R I_{a b c}+\dot{\lambda}_{a b c} \\
\lambda_{a b c}=L_{a b c} I_{a b c}+\Lambda_{r}
\end{array}\right.
$$

where $V_{a b c}, I_{a b c}, \lambda_{a b c}$ and $\Lambda_{r}$ are the stator phase voltages, phase currents, stator fluxes and the rotor permanent magnets flux linkage with the stator windings. $\mathrm{R}$ is a diagonal matrix with $R_{s}$ on the diagonal, $L_{a b c}$ and $\Lambda_{r}$ are reported in (2) and (3) respectively. $\lambda_{m}$ is the peak flux linkage established by the rotor permanent magnets.

$\theta$ is the rotor angular position of the machine measured as the displacement of the quadrature axis (q) from the magnetic axis of phase as. The direct axis (d) is lagging of $90^{\circ}$ behind the $\mathrm{q}$ axis. Transforming the machine phase voltages equations and the stator fluxes of (2) on the rotor synchronous reference frame the following system is obtained:

$$
\left\{\begin{array}{l}
V_{q}=R_{s} i_{q}+\omega \lambda_{d}+\dot{\lambda}_{q} \\
V_{d}=R_{s} i_{d}-\omega \lambda_{q}+\dot{\lambda}_{d} \\
\lambda_{q}=L_{q} i_{q} \\
\lambda_{d}=L_{d} i_{d}+\lambda_{m}
\end{array}\right.
$$

where $\omega$ is the machine electrical speed, $L_{q}$ and $L_{d}$ are the $\mathrm{q}$ and $d$ axes inductances respectively. The relationship between $L_{A}, L_{B}$ and the machine's inductances $L_{q}$ and $L_{d}$ in the synchronous reference frame is reported in (5).

$$
L_{A}=\frac{L_{d}+L_{q}}{3} ; L_{B}=\frac{L_{d}-L_{q}}{3}
$$

In order to digitally implement the model based controller, the discretization of the plant equations is necessary. Firstly the system (4) is written in the standard state space form as

$$
\left[\begin{array}{c}
\dot{i}_{q} \\
\dot{i}_{d}
\end{array}\right]=A\left[\begin{array}{c}
i_{q} \\
i_{d}
\end{array}\right]+B\left[\begin{array}{c}
V_{q} \\
V_{d}
\end{array}\right]+\left[\begin{array}{c}
-\frac{\omega \lambda_{m}}{L_{q}} \\
0
\end{array}\right]
$$

where

$$
A=\left[\begin{array}{cc}
-\frac{R_{s}}{L_{q}} & -\frac{L_{d} \omega}{L_{q}} \\
\frac{L_{q} \omega}{L_{d}} & -\frac{R_{s}}{L_{d}}
\end{array}\right] B=\left[\begin{array}{cc}
\frac{1}{L_{q}} & 0 \\
0 & \frac{1}{L_{d}}
\end{array}\right]
$$

The discretized state-space form of the plant (6) around a generic time instant $\mathrm{k}$ is presented in (7).

$$
\left[\begin{array}{l}
i_{k+1}^{q} \\
i_{k+1}^{d}
\end{array}\right]=\Phi\left[\begin{array}{l}
i_{k}^{q} \\
i_{k}^{d}
\end{array}\right]+\Gamma\left[\begin{array}{c}
V_{k}^{q} \\
V_{k}^{d}
\end{array}\right]+\left[\begin{array}{c}
-\frac{\omega_{k} \lambda_{m}}{L_{q}} \\
0
\end{array}\right]
$$

where

$$
\Phi=e^{A T_{s}} ; \quad \Gamma=\int_{0}^{T_{s}} e^{A \tau} d \tau
$$

Considering the Taylor expansion of $\Phi$ and $\Gamma$ and ignoring the quadratic and higher-order terms the final matrices of the PMSM state-space model used to synthesize the controller are obtained

$$
\Phi=\left[\begin{array}{cc}
1-\frac{R_{s} T_{s}}{L_{q}} & -\frac{\omega_{k} L_{d} T_{s}}{L_{q}} \\
\frac{\omega_{k} L_{q} T_{s}}{L_{d}} & 1-\frac{R_{s} T_{s}}{L_{d}}
\end{array}\right] \Gamma=\left[\begin{array}{cc}
\frac{T_{s}}{L_{q}} & 0 \\
0 & \frac{T_{s}}{L_{d}}
\end{array}\right]
$$

\section{STANDARD DEAD-BEAT CONTROL}

The standard Dead-Beat control can be obtained from the discretized plant equations (7) and (8). By substituting the desired q, d current references to the $i_{k+1}^{q}$ and $i_{k+1}^{d}$ terms and solving for the voltages $V_{k}^{q}$ and $V_{k}^{d}$

$$
\left[\begin{array}{l}
V_{k}^{q} \\
V_{k}^{d}
\end{array}\right]=\Gamma^{-1}\left\{\left[\begin{array}{l}
i_{k+1}^{q R e f} \\
i_{k+1}^{d R e f}
\end{array}\right]-\Phi\left[\begin{array}{l}
i_{k}^{q} \\
i_{k}^{d}
\end{array}\right]-\left[\begin{array}{c}
-\frac{\omega_{k} \lambda_{m}}{L_{q}} \\
0
\end{array}\right]\right\}
$$

Note that the reference value $i_{k+1}^{R e f}$ is needed, but can be assumed to be equal to $i_{k}^{\operatorname{Ref}}$ since $T_{s}$ is sufficiently small compared with the time constant of the system. Therefore the reference can be considered constant over $T_{s}$. The $\mathrm{q}$, d voltage control actions calculated in (9) are the one that, if no saturation occurs, permit to reach the current references in one sample time $T_{s}$. The digital implementation of the standard DB control is possible only if the control action calculation time is negligible respect to the system sample time. Often this condition is not satisfied for standard microcontroller implementations leading to the necessity of taking into account one sample time delay as explained in [1]. The delay can be neglected in a real-time application only if the computational time is significantly reduced to be negligible compared to $T_{s}$.

$$
\begin{gathered}
L_{a b c}=\left[\begin{array}{ccr}
L_{l s}+L_{A}-L_{B} \cos (2 \theta) & -\frac{1}{2} L_{A}-L_{B} \cos 2\left(\theta-\frac{\pi}{3}\right) & -\frac{1}{2} L_{A}-L_{B} \cos 2\left(\theta+\frac{\pi}{3}\right) \\
-\frac{1}{2} L_{A}-L_{B} \cos 2\left(\theta-\frac{\pi}{3}\right) & L_{l s}+L_{A}-L_{B} \cos 2\left(\theta-\frac{2 \pi}{3}\right) & -\frac{1}{2} L_{A}-L_{B} \cos 2(\theta+\pi) \\
-\frac{1}{2} L_{A}-L_{B} \cos 2\left(\theta+\frac{\pi}{3}\right) & -\frac{1}{2} L_{A}-L_{B} \cos 2(\theta+\pi) & L_{l s}+L_{A}-L_{B} \cos 2\left(\theta+\frac{2 \pi}{3}\right)
\end{array}\right] \\
\Lambda_{r}=\lambda_{m}\left[\begin{array}{lll}
\sin (\theta) & \sin \left(\theta-\frac{\pi}{3}\right) & \sin \left(\theta+\frac{\pi}{3}\right)
\end{array}\right]
\end{gathered}
$$




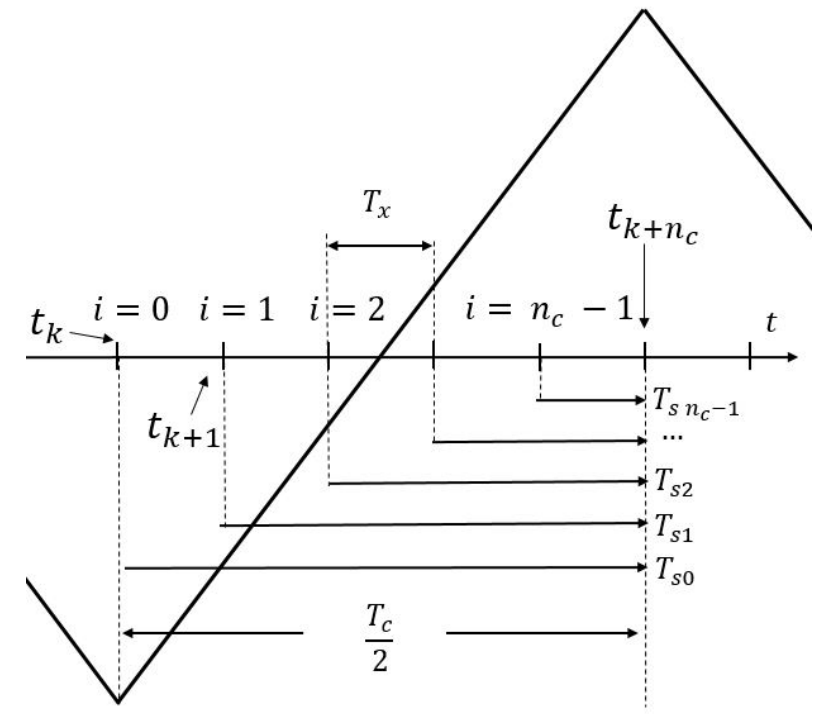

Fig. 1. Carrier and variable sample time for the OS-DB.

\section{OVERSAmpling DeAd-BeAt CONTROL}

The new DB approach here proposed operates at an increased controller sampling frequency without increasing the commutation frequency of the power converter. Referring to Fig. $1, T_{x}$ is the controller sampling period while $T_{c}$ is the modulator carrier period. In order to increase the controller sampling time $T_{x}$ without changing the switching frequency (i.e. the modulator carrier frequency) some consideration should be done. If the control approach described earlier is simply implemented to a higher frequency, the system becomes quickly unstable as the ratio $\frac{T_{x}}{T_{c}}$ decreases due to the interaction with the modulator. The control voltages (9) in fact, are optimal only if they are equal to the inverter output voltages averaged on a $T_{x}$ period. Using a modulator however, this is guaranteed only on a carrier semi-period, i.e. $\frac{T_{c}}{2}$. The oversampling coefficient $n_{c}$ is defined as

$$
n_{c}=\frac{T_{c}}{2 T_{x}}
$$

Fig. 1 shows a variable sample time $T_{s}(i)$ that depends on the oversampling coefficient $n_{c}$ and the modulator triangular carrier period and it is defined as

$$
T_{s}(i)=\frac{T_{c}}{2}-i T_{x}=\left(n_{c}-i\right) T_{x}
$$

where $i=0,1,2, \ldots, n_{c}-1$ and it is reset to 0 every $\frac{T_{c}}{2}$, i.e. at the end of each carrier segment. In order to take into account the variable sample time of the controller, (11) can be substituted to the discretized plant equations (7) and (8) to obtain

$$
\begin{array}{r}
{\left[\begin{array}{c}
i_{k+n_{c}}^{q} \\
i_{k+n_{c}}^{d}
\end{array}\right]=\Phi_{T_{s}(i)}\left[\begin{array}{c}
i_{k+i}^{q} \\
i_{k+i}^{d}
\end{array}\right]+} \\
\Gamma_{T_{s}(i)}\left[\begin{array}{c}
V_{k+i}^{q} \\
V_{k+i}^{d}
\end{array}\right]+\left[\begin{array}{c}
-\frac{\omega_{k+i} \lambda_{m}}{L_{q}} \\
0
\end{array}\right]
\end{array}
$$

The matrices $\Phi_{T_{s}(i)}$ and $\Gamma_{T_{s}(i)}$ are identical to (8) where $T_{s}$ has been substituted with (11). Please observe that the time subscript $k$ of (12), according to Fig. 1, increases every $T_{x}$ by 1 according to the new oversampling coefficient $n_{c}$. The DBOS controller output voltages are obtained from (12) resolving for $V_{k+i}^{q}$ and $V_{k+i}^{d}$

$$
\begin{aligned}
{\left[\begin{array}{c}
V_{k+i}^{q} \\
V_{k+i}^{d}
\end{array}\right]=\Gamma_{T_{s}(i)}^{-1}\left\{\left[\begin{array}{l}
i_{k+n_{c}}^{q} \\
i_{k+n_{c}}^{d}
\end{array}\right]-\right.} \\
\left.\Phi_{T_{s}(i)}\left[\begin{array}{c}
i_{k+i}^{q} \\
i_{k+i}^{d}
\end{array}\right]-\left[\begin{array}{c}
-\frac{\omega_{k+i} \lambda_{m}}{L_{q}} \\
0
\end{array}\right]\right\}
\end{aligned}
$$

Therefore the controller voltages $k+n_{c}$ are calculated $n_{c}$ times, where each time the sample time is reduced by $T_{x}$. As already mentioned, one of the main problems of predictive controllers is the strong dependence by the model parameters. In fact, in real applications, both the stator resistance and inductance can vary causing a significant degradation in the DB controller performances. The oversampled technique investigated allows to compute the controller equations with higher frequency therefore reducing the effect of incorrect predictions due to parameters variation from the rated ones.

\section{A. Voltage distortion compensation}

It can be seen in Section III the standard DB current loop controller is able to achieve one sample time dynamic response to a variation in current references if no variation of the PMSM parameters has happened. In the OS-DB controller voltages are computed every $\frac{T_{c}}{2 n_{c}}$ with a reducing sample time. For a standard controller that operates at a frequency equal or double the converter switching frequency, it is true that the controller voltage computed will be equal to the average voltage applied by the converter over a sample time. Instantaneously, this relationship is not true, in fact due to their intrinsic discrete nature, power converters are able to output only a finite number of the voltage. For a standard three phase two level Voltage Source Converter (2L-VSI) the phase voltages can be equal to $\frac{V_{d} c}{2}$ or $\frac{-V_{d} c}{2}$ and they are modulated according to PWM principle to achieve, on average, the reference voltage. OSDB controller runs $n_{c}$ times over half of a carrier period. It means that the reference voltage generated cannot be applied, not even on average, in a single controller sample time $T_{x}$. For this reason, a compensation term must be added to (13).

According to the PMSM model the qd currents at the time instant $k+i$ can be predicted from the previous time instant $k+i-1$ as

$$
\begin{array}{r}
{\left[\begin{array}{l}
i_{k+i}^{q} \\
i_{k+i}^{d}
\end{array}\right]=\Phi_{T_{x}}\left[\begin{array}{l}
i_{k+i-1}^{q} \\
i_{k+i-1}^{d}
\end{array}\right]+} \\
\Gamma_{T_{x}}\left[\begin{array}{c}
V_{k+i-1}^{q C} \\
V_{k+i-1}^{d C}
\end{array}\right]+\left[\begin{array}{c}
-\frac{\omega_{k+i-1} \lambda_{m}}{L_{q}} \\
0
\end{array}\right]
\end{array}
$$




$$
\begin{aligned}
& {\left[\begin{array}{l}
i_{k+i}^{q} \\
i_{k+i}^{d}
\end{array}\right]=\Phi_{T_{x}}\left[\begin{array}{l}
i_{k+i-1}^{q} \\
i_{k+i-1}^{d}
\end{array}\right]+} \\
& \Gamma_{T_{x}}\left[\begin{array}{l}
V_{k+i-1}^{q R} \\
V_{k+i-1}^{d R}
\end{array}\right]+\left[\begin{array}{c}
-\frac{\omega_{k+i-1} \lambda_{m}}{L_{q}} \\
0
\end{array}\right]
\end{aligned}
$$

where the PMSM matrices $\Phi_{T_{x}}$ and $\Gamma_{T_{x}}$ correspond to (8), but discretized over a time $T_{x}$. Equations (14) and (15) predict motor currents at time instant $k+i$ assuming at the previous sample time $k+i-1$ has been applied the controller voltage $V_{k+i-1}^{C}$ or the real voltage $V_{k+i-1}^{R}$ respectively. By replacing (14) and (15) in (13) the controller voltages at the time instant $k+i$ in dependence of $V_{k+i-1}^{C}$ and $V_{k+i-1}^{R}$ are obtained as in (18) and (19).

A compensation terms for both $\mathrm{q}$ and $\mathrm{d}$ axis can be computed subtracting (19) to (18)

$$
\begin{aligned}
{\left[\begin{array}{c}
V_{c k+i}^{q} \\
V_{c_{k+i}}^{d}
\end{array}\right]=} & {\left[\begin{array}{cc}
\frac{R_{s} T_{x}}{L_{q}}+\frac{1}{i-n_{c}} & w_{k+i} T_{x} \\
-w_{k+i} T_{x} & \frac{R_{s} T_{x}}{L_{d}}+\frac{1}{i-n_{c}}
\end{array}\right] } \\
& {\left[\begin{array}{l}
V_{k+i-1}^{q C}-V_{k+i-1}^{q R} \\
V_{k+i-1}^{d C}-V_{k+i-1}^{d R}
\end{array}\right] }
\end{aligned}
$$

The final equations of the OS-DB controller are the following

$$
\begin{gathered}
{\left[\begin{array}{c}
V_{k+i}^{q} \\
V_{k+i}^{d}
\end{array}\right]=\Gamma_{T_{s}(i)}^{-1}\left\{\left[\begin{array}{c}
i_{k+n_{c}}^{q} \\
i_{k+n_{c}}^{d}
\end{array}\right]-\Phi_{T_{s}(i)}\left[\begin{array}{c}
i_{k+i}^{q} \\
i_{k+i}^{d}
\end{array}\right]-\right.} \\
\left.\left[\begin{array}{c}
-\frac{\omega_{k+i} \lambda_{m}}{L_{q}} \\
0
\end{array}\right]\right\}+\left[\begin{array}{c}
V_{c_{k+i}}^{q} \\
V_{c_{k+i}}^{d}
\end{array}\right]
\end{gathered}
$$

\section{B. Real Voltages Calculation and OS-DB Controller Imple- mentation}

As it can be seen from (16) in order to compute the compensation terms for the OS-DB it is necessary to know both the controller and real voltages applied at the previous time instant. The controller voltages $V_{k+i-1}^{C}$ can be stored in memory in order to be used at the actual time instant while the real voltages $V_{k+i-1}^{R}$ applied by the converter need to be computed. Fig. 2 shows the standard Pulse Width Modulation
TABLE I

MODEL PARAMETERS.

\begin{tabular}{|c|c||c|c|}
\hline$L_{q}$ & $4.1[\mathrm{mH}]$ & $L_{d}$ & $2.58[\mathrm{mH}]$ \\
\hline$R_{s}$ & $1.35[\Omega]$ & $p$ & $4[-]$ \\
\hline$J_{m}$ & $31.685\left[\mathrm{~g} \mathrm{~m}^{2}\right]$ & $\omega_{m}$ rated & $150\left[\frac{\text { rad }}{s}\right]$ \\
\hline$V_{d c}$ & $300[\mathrm{~V}]$ & $D T$ & $2.2[\mu s]$ \\
\hline$f_{s w}$ & $10[\mathrm{KHz}]$ & $T_{c}$ & $100[\mu s]$ \\
\hline$T_{x}$ & $10[\mu s]$ & $n_{c}$ & $5[-]$ \\
\hline
\end{tabular}

(PWM) for a generic phase $j$ and the correspondent converter leg voltage. The knowledge of the carrier and the phase reference signal are enough to calculate the phase voltage. Knowing the time instant $t_{s w}$ at which the modulating voltage crosses with the carrier the average voltage applied by the converter can be calculated as follows

$$
\bar{V}_{j}=\frac{V_{d c}}{2} \frac{t_{s w}}{T_{s}}-\frac{V_{d c}}{2}\left(1-\frac{t_{s w}}{T_{s}}\right)
$$

\section{Disturbance ObServer}

The performances of the DB control such as the dynamic behaviour and steady state offset are strongly dependant on the knowledge of the plant model and its parameters. Any mismatch in the modelization of the plant or uncertainty in the parameter will lead to a deterioration of the performances. It can be noticed from (9) and (17) that both for the DB and for the OS-DB control there is no integral action. Therefore any uncertainty in the stator resistance, flux established by the rotor permanent magnets, inverter non-linearities and influence of external disturbances would lead to a current steady state offset. To avoid this problem a reduced order estimator for the voltage disturbances has been modelled and implemented. The overall control block diagram with the reduced order Luenberger disturbance estimator is shown in Fig. 3. Assuming the disturbances as constants the continuous estimator equations are

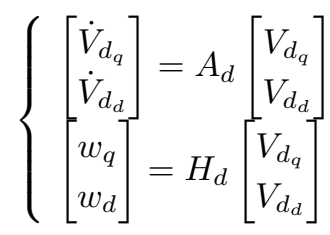

where

$$
A_{d}=\left[\begin{array}{ll}
0 & 0 \\
0 & 0
\end{array}\right] ; \quad H_{d}=\left[\begin{array}{ll}
1 & 0 \\
0 & 1
\end{array}\right]
$$

$$
\begin{aligned}
& {\left[\begin{array}{l}
V_{k+i}^{q C} \\
V_{k+i}^{d C}
\end{array}\right]=\Gamma_{T_{s}(i)}^{-1}\left\{\left[\begin{array}{c}
i_{k+n_{c}}^{q} \\
i_{k+n_{c}}^{d}
\end{array}\right]-\Phi_{T_{s}(i)}\left[\Phi_{T_{x}}\left[\begin{array}{l}
i_{k+i-1}^{q} \\
i_{k+i-1}^{d}
\end{array}\right]+\Gamma_{T_{x}}\left[\begin{array}{l}
V_{k+i-1}^{q C} \\
V_{k+i-1}^{d C}
\end{array}\right]+\left[\begin{array}{c}
-\frac{\omega_{e k+i-1} \lambda_{m}}{L_{q}} \\
0
\end{array}\right]\right]-\left[\begin{array}{c}
-\frac{\omega_{e k+i} \lambda_{m}}{L_{q}} \\
0
\end{array}\right]\right\}} \\
& {\left[\begin{array}{l}
V_{k+i}^{q R} \\
V_{k+i}^{d R}
\end{array}\right]=\Gamma_{T_{s}(i)}^{-1}\left\{\left[\begin{array}{c}
i_{k+n_{c}}^{q} \\
i_{k+n_{c}}^{d}
\end{array}\right]-\Phi_{T_{s}(i)}\left[\Phi_{T_{x}}\left[\begin{array}{l}
i_{k+i-1}^{q} \\
i_{k+i-1}^{d}
\end{array}\right]+\Gamma_{T_{x}}\left[\begin{array}{l}
V_{k+i-1}^{q R} \\
V_{k+i-1}^{d R}
\end{array}\right]+\left[\begin{array}{c}
-\frac{\omega_{e k+i-1} \lambda_{m}}{L_{q}} \\
0
\end{array}\right]\right]-\left[\begin{array}{c}
-\frac{\omega_{e k+i} \lambda_{m}}{L_{q}} \\
0
\end{array}\right]\right\}}
\end{aligned}
$$




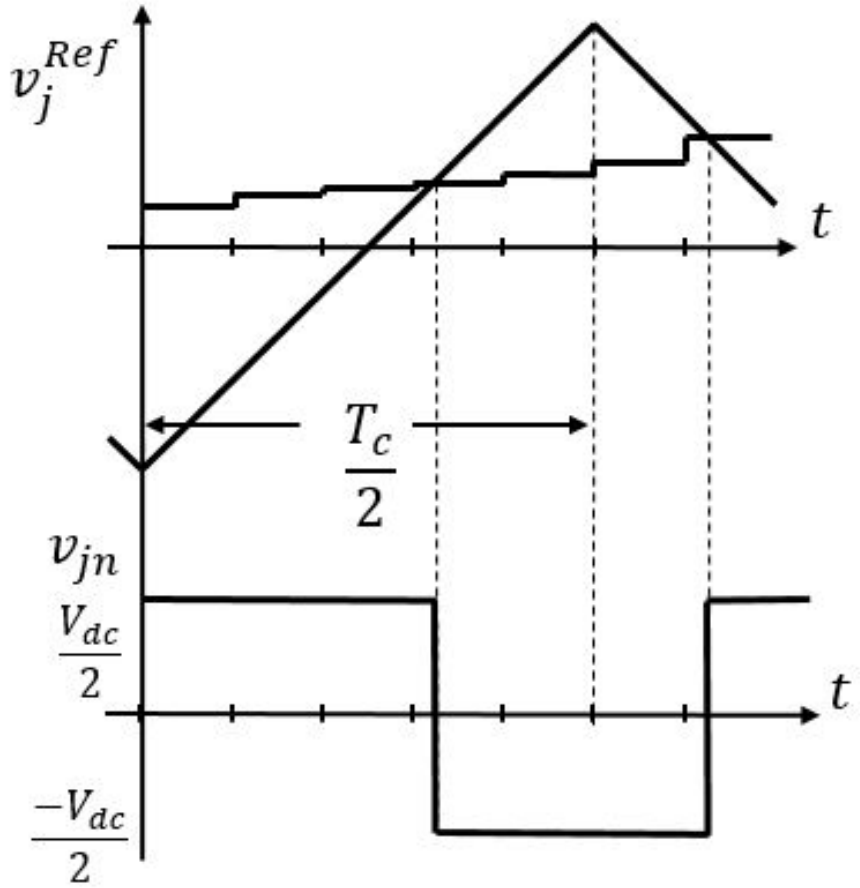

Fig. 2. PWM and phase leg voltage.

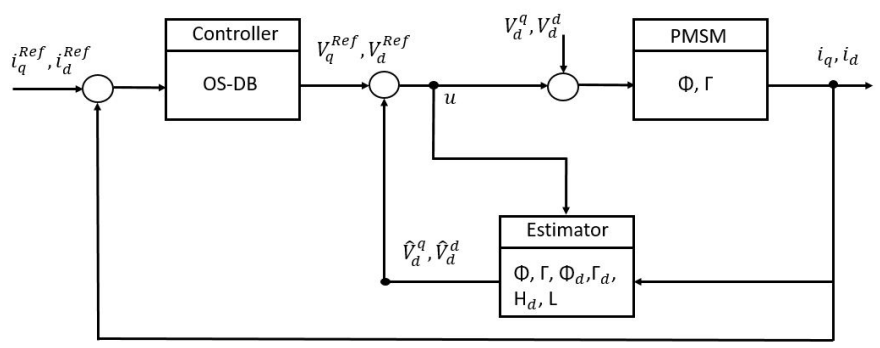

Fig. 3. Block scheme of the complete control structure.

and where $V_{d_{q}}$ and $V_{d_{d}}$ are the voltage disturbances. The discrete state space form of the disturbance equations are

$$
\left[\begin{array}{c}
V_{d_{k+1}}^{q} \\
V_{d_{k+1}}^{d}
\end{array}\right]=\Phi_{d}\left[\begin{array}{c}
V_{d_{k}}^{q} \\
V_{d_{k}}^{d}
\end{array}\right]
$$

where

$$
\Phi_{d}=\left[\begin{array}{ll}
1 & 0 \\
0 & 1
\end{array}\right]
$$

The plant discretized model in the state space form considering the voltage disturbances are

$$
\left[\begin{array}{l}
i_{k+1}^{q} \\
i_{k+1}^{d}
\end{array}\right]=\Phi\left[\begin{array}{l}
i_{k}^{q} \\
i_{k}^{d}
\end{array}\right]+\Gamma\left[\begin{array}{c}
V_{k}^{q} \\
V_{k}^{d}
\end{array}\right]+\left[\begin{array}{c}
-\frac{\omega_{k} \lambda_{m}}{L_{q}} \\
0
\end{array}\right]+\Gamma_{d}\left[\begin{array}{c}
V_{d_{k}}^{q} \\
V_{d_{k}}^{d}
\end{array}\right]
$$

where

$$
\Gamma_{d}=\left[\begin{array}{cc}
-\frac{T_{s}}{L_{q}} & 0 \\
0 & -\frac{T_{s}}{L_{d}}
\end{array}\right]
$$

The state of the plant is augmented by two by taking into account the disturbances as follows

$$
\left\{\begin{array}{l}
x_{k+1}=\left[\begin{array}{cc}
\Phi & \Gamma_{d} H_{d} \\
0 & \Phi_{d}
\end{array}\right] x_{k}+\left[\begin{array}{c}
\Gamma \\
0
\end{array}\right] u_{k} \\
{\left[\begin{array}{c}
i_{k}^{q} \\
i_{k}^{d}
\end{array}\right]=\left[\begin{array}{llll}
1 & 0 & 0 & 0 \\
0 & 1 & 0 & 0
\end{array}\right] x_{k}}
\end{array}\right.
$$

where

$$
x_{k}=\left[\begin{array}{llll}
i_{k}^{q} & i_{k}^{d} & V_{d_{k}}^{q} & V_{d_{k}}^{d}
\end{array}\right]^{T} ; \quad u_{k}=\left[\begin{array}{cc}
V_{k}^{q} & V_{k}^{q}
\end{array}\right]^{T}
$$

Finally the reduced order Luenberger estimator has been implemented for the augmented state plant equations (24) obtaining

$$
\begin{aligned}
& {\left[\begin{array}{c}
\hat{V}_{d_{k}}^{q} \\
\hat{V}_{d_{k}}^{d}
\end{array}\right]=\left(\Phi_{d}-L_{r} \Gamma_{d} H_{d}\right)\left[\begin{array}{l}
\hat{V_{d_{k-1}}^{q}} \\
\hat{V}_{d_{k-1}}^{d}
\end{array}\right]} \\
& +L_{r}\left(\left[\begin{array}{l}
i_{k}^{q} \\
i_{k}^{d}
\end{array}\right]-\Phi\left[\begin{array}{l}
i_{k-1}^{q} \\
i_{k-1}^{d}
\end{array}\right]-\Gamma u_{k-1}\right)
\end{aligned}
$$

where $L_{r}$ is a $2 \times 2$ matrix representing the Luenberger gain which has to be calculated in order to obtain the desired estimator dynamic.

\section{Simulations Results}

The proposed method has been firstly analyzed in simulation using Matlab-Simulink and the Xilinx System Generator toolbox in order to test the FPGA code in closed loop with the entire system. The overall control structure implemented is presented in Fig. 3. The OS-DB block implements the controller described in Section IV and IV-A while the estimator block implements the equations described in Section V. The proposed OS-DB controller is not affected by the control strategy used to regulate the motor speed. Therefore, for clarity and simplicity, a standard proportional integral (PI) controller has been used for the speed loop. Furthermore since the focus of this work are the current loop performances, no current profiling technique like Maximum Torque Per Ampere (MTPA) has been implemented and the torque is generated using only $i_{q}$, while $i_{d}$ is controlled to zero. As reported in [21] the recursive least square method has been used to estimate both the electrical and mechanical parameters of the motor. The parameters used to simulate the model correspond to the real ones and are reported in Table I. The proposed oversampled predictive controller measures and computes every $T_{x}$ but actuates only once every $\frac{T_{c}}{2}$. Considering a PMSM drive with controller sample time of $50 \mu \mathrm{s}$, converter switching frequency of $10 \mathrm{KHz}$ and an oversampling coefficient $n_{c}$ of 5 then the sampling period $T_{x}$ of the OS-DB controller is $10 \mu \mathrm{s}$.

Firstly the performances of the OS-DB controller are compared with the standard DB controller when no parameter variation occurs. Fig. 4 shows the dynamic behaviour in response 


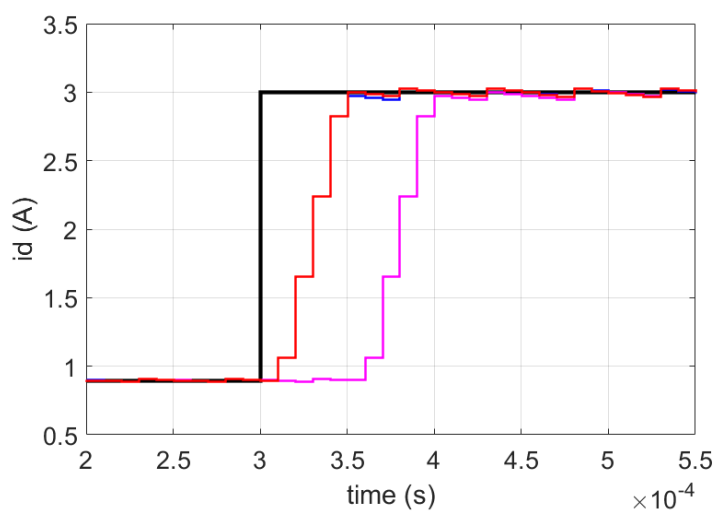

Fig. 4. System response to a reference $i_{d}$ step from 0.9 to 3 A. reference current $i_{d}$ (black line), DSP DB $i_{d}$ (magenta line), FPGA DB $i_{d}$ (blue line), OS-DB $i_{d}$ (red line).

to a $i_{d}$ step from 0.9 to $3 \mathrm{~A}$ at standstill of three different controllers: the standard DB controller implemented in DSP (with the step delay compensation), the FPGA implemented DB and the OS-DB, also FPGA implemented. It can be noticed how the DSP DB controller response is one sampling time $50 \mu \mathrm{s}$ slower compared to the other controllers. The ideal current response with only one sample time delay is achieved for both the FPGA DB and for the new proposed OS-DB controller (superimposed red and blue lines in Fig. 4). Since intrinsically the performances of the DSP implemented DB are inferior to the other controllers, it will not anymore be considered in the following. The voltage reference distortion introduced by the uncompensated OS-DB and the dynamic performances degradation are shown in Fig. 5 and Fig. 6 respectively. It is evident how the introduced compensation result to be necessary if low distortion and fast transient response want to be achieved. The main advantage of the proposed controller is the ability to achieve better transient performances when a machine parameter variation happens. The transient and steady state performances are evaluated for an $L_{d}$ reduction and increment from the rated parameter of $50 \%$. Fig. 7 shows how the OS-DB maintains the same transient performances even when a significant parameter variation occurs. Fig. 8 shows instead how the phase current profile remains unchanged when the controller operates with and without parameter variation. It is clear how the proposed controller significantly improves the transient response under parameters uncertainties while not degrading the steady state behaviour. In Section VII a more in depth analysis of the controller performances under different working conditions is carried out.

\section{EXPERIMENTAL RESULTS}

The proposed method has been finally tested on an experimental set-up composed by a PMSM coupled with a DC motor as shown in Fig. 9. The PMSM is fed by a two level IGBT converter while the control board specification can be found in [22]. The overall control performances have been tested under different operating conditions for two cases: rated parameters and parameters variation.
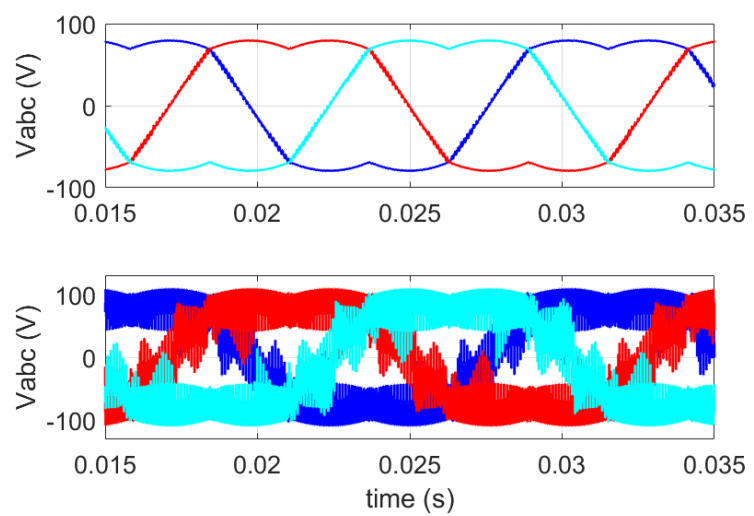

Fig. 5. OS-DB voltage references. Top panel: compensated OS-DB. Bottom Panel: uncompensated OS-DB.

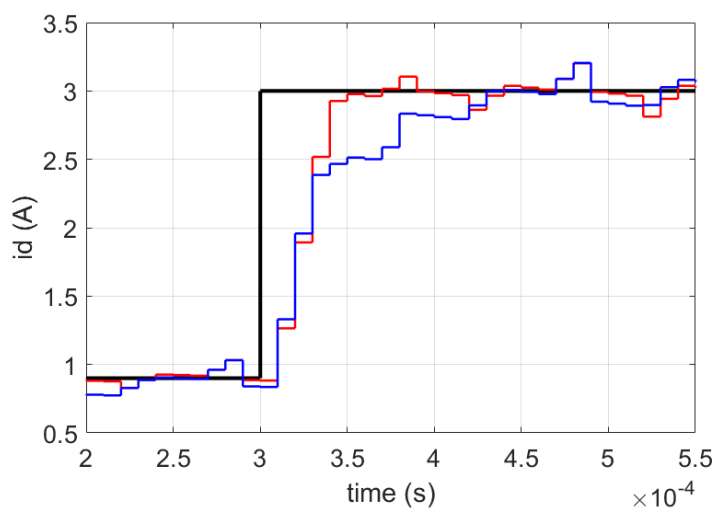

Fig. 6. OS-DB response to a reference $i_{d}$ step from 0.9 to 3 A. Reference current $i_{d}$ (black line), uncompensated OS-DB $i_{d}$ (blue line), compensated OS-DB $i_{d}$ (red line).

\section{A. Rated Parameters}

When the OS-DB predictive controller parameters coincide with the PMSM ones it can be referred to as the rated parameter case. Fig. 10 shows how the same performances expected in simulation are obtained on the experimental setup for an $i_{d}$ step from 0.9 to $3 \mathrm{~A}$ at standstill. The transient response of the OS-DB is equivalent to the one of the FPGA implemented DB. Fig. 11 shows the same $i_{d}$ transient comparison of performances of the DB FPGA implemented and the OS-DB at a mechanical speed $\omega_{m}$ of $150 \frac{\mathrm{rad}}{\mathrm{s}}$. The controller performances on the quadrature axis are evaluated controlling the speed with the load dc motor and asking for an $i_{q}$ step on the PMSM. Fig. 12 shows a $i_{q}$ step from 0.9 to $3 \mathrm{~A}$ at $\omega_{m}$ of $130 \frac{\mathrm{rad}}{\mathrm{s}}$.

\section{B. Parameters Variation}

As already mentioned the main problem of predictive controllers is the strong dependency of the controller from the model parameters. In order to verify the robustness of the proposed controller to parameters uncertainties an experimental analysis has been carried out. In a real application both the stator resistance and the inductance can vary. The first 

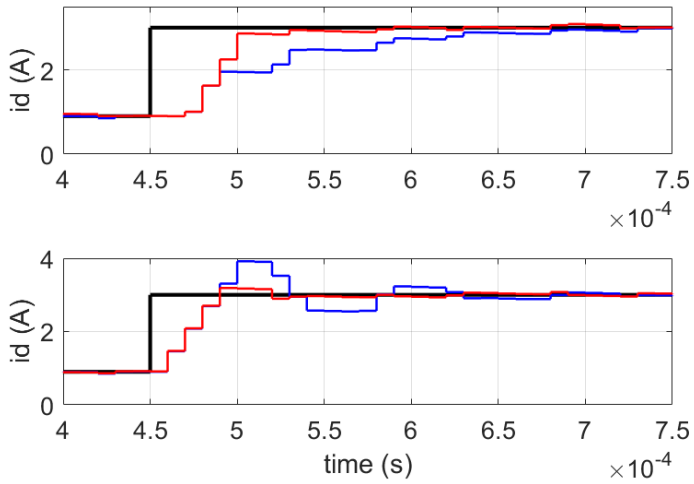

Fig. 7. OS-DB $i_{d}$ step from 0.9 to $3 \mathrm{~A}$ at $\omega_{m} 50 \stackrel{\mathrm{rad}}{ }$. Top panel: $50 \%$ $L_{d}$ increment. Reference current $i_{d}$ (black line), DB FPSGA implemented $i_{d}$ (blue line), OS-DB $i_{d}$ (red line). Bottom Panel: $50 \% L_{d}$ reduction. Reference current $i_{d}$ (black line), DB FPGA implemented $i_{d}$ (blue line), OS-DB $i_{d}$ (red line).
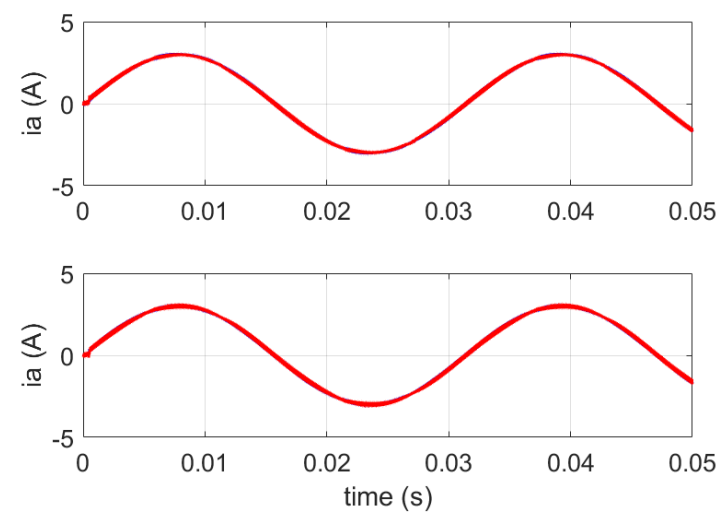

Fig. 8. OS-DB phase current steady state performances at $\omega_{m} 50 \frac{\mathrm{rad}}{\mathrm{s}}$. Top panel: $50 \% L_{d}$ increment. DB FPGA implemented $i_{a}$ (blue line), OS $\stackrel{S}{\mathrm{~S}}$-DB $i_{a}$ (red line). Bottom Panel: $50 \% L_{d}$ reduction. DB FPGA implemented $i_{a}$ (blue line), OS-DB $i_{a}$ (red line).

one is mainly affected by temperature with an uncertainty up to $30 \%$ while the latter can change due to ferromagnetic saturation. Variation in stator resistance or flux established by the permanent magnets mainly affects the steady state performances of the controller but since a disturbance observer has been implemented any possible steady state offset would be cancelled. In order to emulate the saturation of the ferromagnetic core the controller inductance is set to be higher of the machine one. In this way the controller will see a lower machine inductance as wanted. On the other hand a controller inductance lower than the machine one could represent the case of poor knowledge of the PMSM parameters. The test, whose results are reported in Fig. 13, has been performed with an increase of the controller $L_{d}$ inductance of $50 \%$ of the nominal value with respect to the PMSM one while Fig. 14 shows the test where a reduction of $50 \%$ has taken place. As it can be noticed the OS-DB achieves a faster transient compared to the standard implemented FPGA DB in both cases of inductance variation. Fig. 15 shows the experimental results for a $i_{q}$ transient derived from an increase of the controller

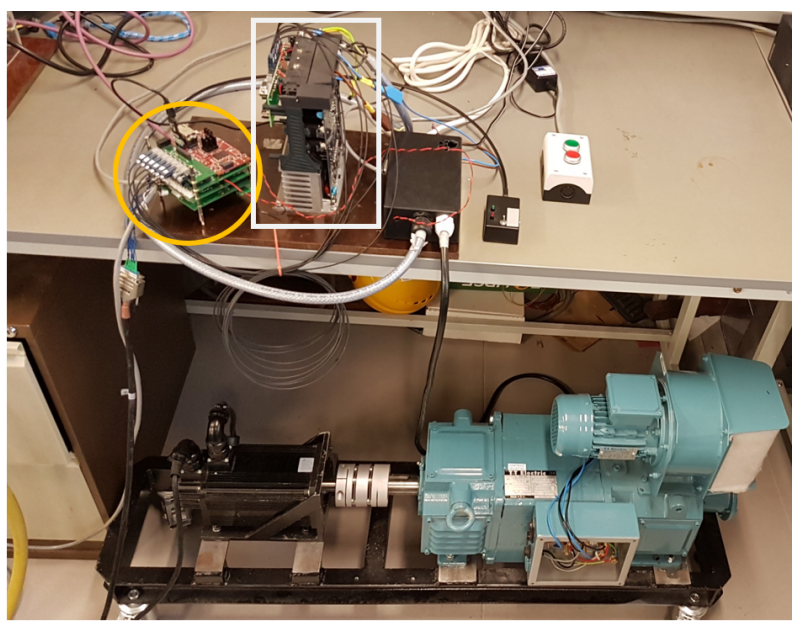

Fig. 9. Experimental set-up: 2L-VSI (white rectangle), control board (yellow circle) and motors.

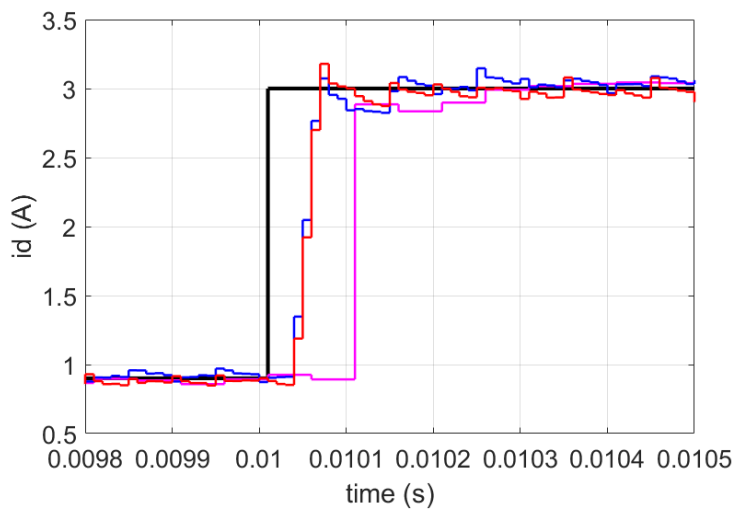

Fig. 10. System response to a reference $i_{d}$ step from 0.9 to $3 \mathrm{~A}$ at standstill. Reference current $i_{d}$ (black line), DSP DB $i_{d}$ (magenta line), FPGA DB $i_{d}$ (blue line), OS-DB $i_{d}$ (red line).

$L_{q}$ inductance of $50 \%$ while Fig. 16 shows the same transient at a different speed and for a reduction of $50 \%$ of the nominal value with respect to the PMSM one. Also for the quadrature axis inductance under any mismatch the OS-DB outperforms the standard FPGA implemented DB.

\section{CONCLUSIONS}

In this paper a novel OS-DB current control approach for PMSMs drives operating at a controller sampling frequency multiple of the power converter switching frequency has been proposed. A disturbance observer has been implemented to estimate the converter voltage distortions. The FPGA implemented OS-DB controller permits to achieve a constant switching frequency and an optimal current ripple along with a high current loop bandwidth and robust behaviour to parameter variation. Both simulative and experimental results show that the proposed controller performs as a standard FPGA implemented DB controller when the controller parameters coincide with the machine parameters. The robustness of the modelbased controller and observer with respect to parameter mismatching has been investigated showing that the performances largely increase when a OS-DB solution is adopted. 


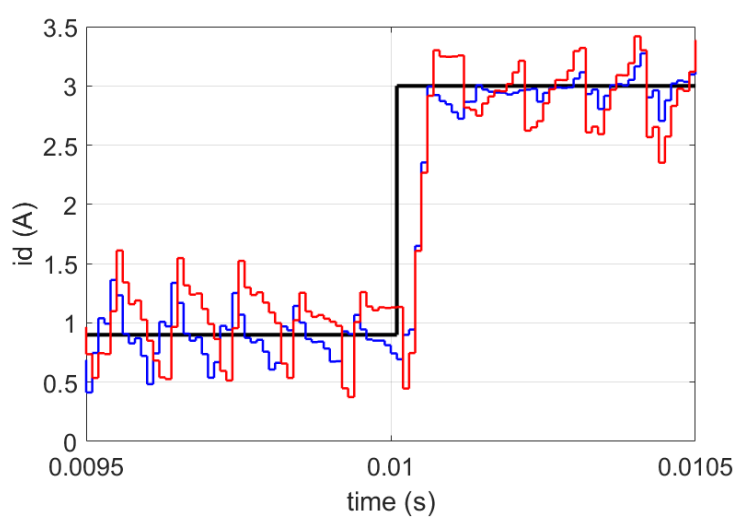

Fig. 11. $i_{d}$ step from 0.9 to $3 \mathrm{~A}$ at $\omega_{m}$ of $150 \frac{\mathrm{rad}}{\mathrm{s}}$. Reference current $i_{d}$ (black line), FPGA DB $i_{d}$ (blue line), OS-DB $i_{d}$ (red line).

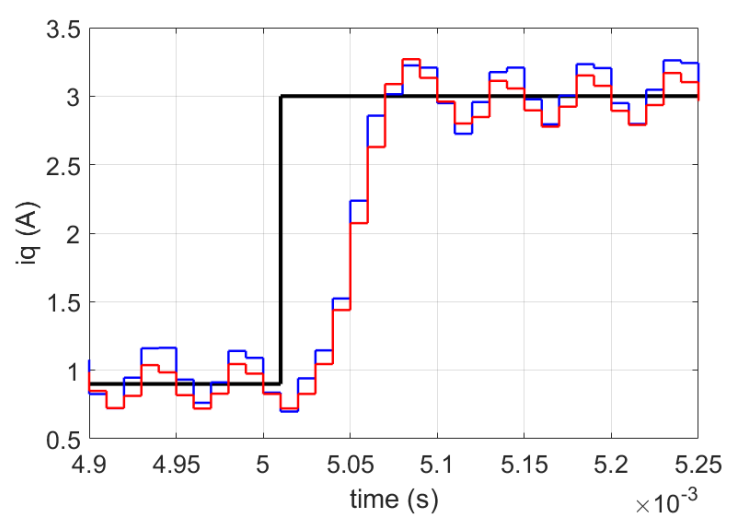

Fig. 12. $i_{q}$ step from 0.9 to $3 \mathrm{~A}$ at $\omega_{m}$ of $130 \frac{\mathrm{rad}}{\mathrm{s}}$. Reference current $i_{q}$ (black line), FPGA DB $i_{q}$ (blue line), OS-DB $i_{q}$ (red line).

\section{REFERENCES}

[1] S. Kouro, P. Cortes, R. Vargas, U. Ammann, and J. Rodriguez, "Model predictive control; a simple and powerful method to control power converters," IEEE Transactions on Industrial Electronics, vol. 56, no. 6 , pp. 1826-1838, June 2009.

[2] J. Rodriguez, M. P. Kazmierkowski, J. R. Espinoza, P. Zanchetta H. Abu-Rub, H. A. Young, and C. A. Rojas, "State of the art of finite control set model predictive control in power electronics," IEEE Transactions on Industrial Informatics, vol. 9, no. 2, pp. 1003-1016, May 2013.

[3] L. Rovere, A. Formentini, A. Gaeta, P. Zanchetta, and M. Marchesoni, "Sensorless finite-control set model predictive control for ipmsm drives," IEEE Transactions on Industrial Electronics, vol. 63, no. 9, pp. 59215931, Sept 2016.

[4] T. Kawabata, T. Miyashita, and Y. Yamamoto, "Dead beat control of three phase pwm inverter," IEEE Transactions on Power Electronics, vol. 5, no. 1, pp. 21-28, Jan 1990.

[5] P. Mattavelli, "An improved deadbeat control for ups using disturbance observers," IEEE Transactions on Industrial Electronics, vol. 52, no. 1, pp. 206-212, Feb 2005.

[6] L. Malesani, P. Mattavelli, and S. Buso, "Robust dead-beat current control for pwm rectifiers and active filters," IEEE Transactions on Industry Applications, vol. 35, no. 3, pp. 613-620, May 1999.

[7] P. Wipasuramonton, Z. Q. Zhu, and D. Howe, "Predictive current control with current-error correction for pm brushless ac drives," IEEE Transactions on Industry Applications, vol. 42, no. 4, pp. 1071-1079, July 2006.

[8] M. Tang, A. Gaeta, K. Ohyama, P. Zanchehtta, and G. Asher, "Assessments of dead beat current control for high speed permanent magnet synchronous motor drives," in 2015 9th International Conference on

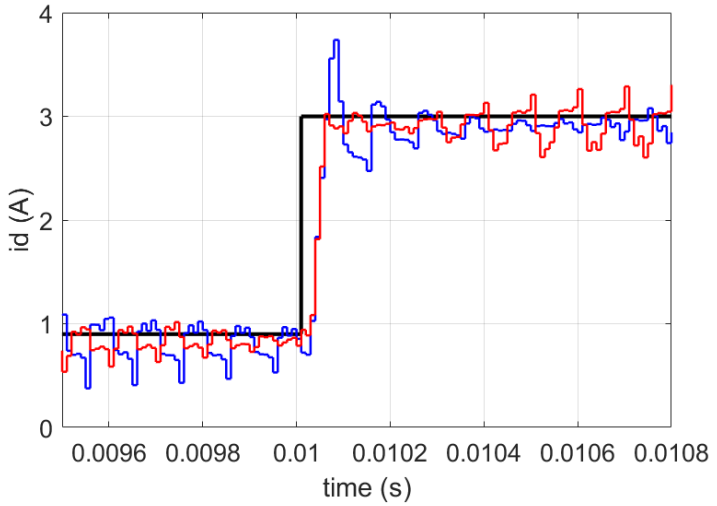

Fig. 13. $i_{d}$ step from 0.9 to $3 \mathrm{~A}$ at $\omega_{m}$ of $100 \frac{\mathrm{rad}}{\mathrm{s}}$. Reference current $i_{d}$ (black line), FPGA DB $i_{d}$ (blue line), OS-DB $i_{d}$ (red line).

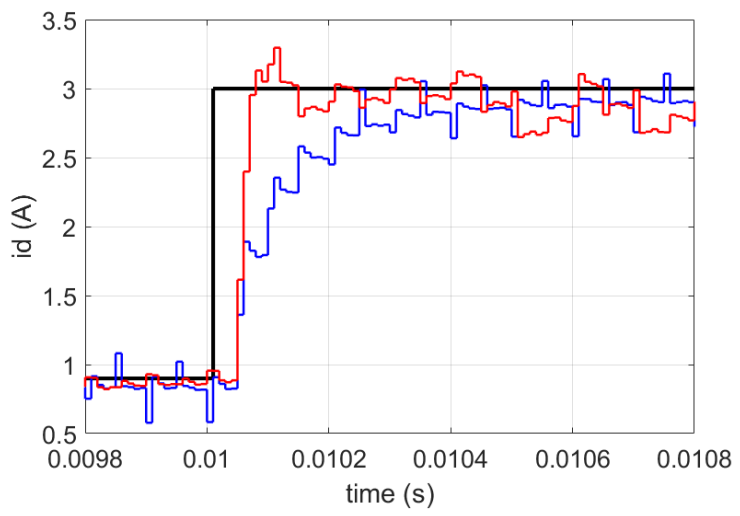

Fig. 14. $i_{d}$ step from 0.9 to $3 \mathrm{~A}$ at $\omega_{m}$ of $50 \frac{\mathrm{rad}}{\mathrm{s}}$. Reference current $i_{d}$ (black line), FPGA DB $i_{d}$ (blue line), OS-DB $i_{d}$ (red line).

Power Electronics and ECCE Asia (ICPE-ECCE Asia), June 2015, pp. 1867-1874.

[9] L. Springob and J. Holtz, "High-bandwidth current control for torqueripple compensation in pm synchronous machines," IEEE Transaction on Industrial Electronics, vol. 45, no. 5, pp. 713-721, Oct 1998.

[10] H.-T. Moon, H.-S. Kim, and M.-J. Youn, "A discrete-time predictive current control for pmsm," IEEE Transactions on Power Electronics, vol. 18, no. 1, pp. 464-472, Jan 2003.

[11] P. Mattavelli, G. Spiazzi, and P. Tenti, "Predictive digital control of power factor preregulators with input voltage estimation using disturbance observers," IEEE Transactions on Power Electronics, vol. 20, no. 1, pp. 140-147, Jan 2005.

[12] O. Gulbudak and E. Santi, "Fpga-based model predictive controller for direct matrix converter," IEEE Transactions on Industrial Electronics, vol. 63, no. 7, pp. 4560-4570, July 2016.

[13] M. W. Naouar, A. A. Naassani, E. Monmasson, and I. Slama-Belkhodja, "Fpga-based predictive current controllerfor synchronous machine speed drive," IEEE Transactions on Power Electronics, vol. 23, no. 4, pp. 2115-2126, July 2008.

[14] M. Curkovic, K. Jezernik, and R. Horvat, "Fpga-based predictive sliding mode controller of a three-phase inverter," IEEE Transactions on Industrial Electronics, vol. 60, no. 2, pp. 637-644, Feb 2013.

[15] B. C. Alecsa and A. Onea, "An fpga implementation of the time domain deadbeat algorithm for control applications," in 2009 NORCHIP, Nov 2009 , pp. 1-4.

[16] K. Miyata, K. Tsuchiya, and T. Yokoyama, "A study of $1 \mathrm{mhz}$ multisampling deadbeat control with disturbance compensation for pmsm drive system using fpga," in 2016 IEEE 8th International Power Electronics and Motion Control Conference (IPEMC-ECCE Asia), May 2016, pp. 654-659.

[17] T. Trker, U. Buyukkeles, and A. F. Bakan, "A robust predictive current 


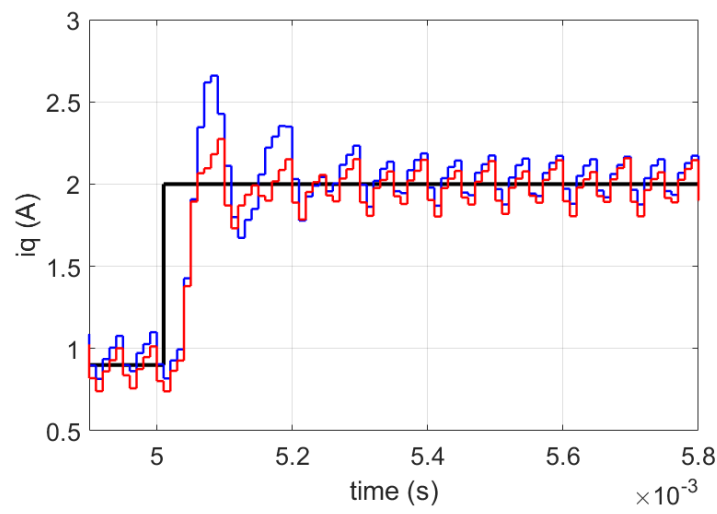

Fig. 15. $i_{q}$ step from 0.9 to $3 \mathrm{~A}$ at $\omega_{m}$ of $50 \frac{\mathrm{rad}}{\mathrm{s}}$. Reference current $i_{q}$ (black line), FPGA DB $i_{q}$ (blue line), OS-DB $i_{q}$ (red line).

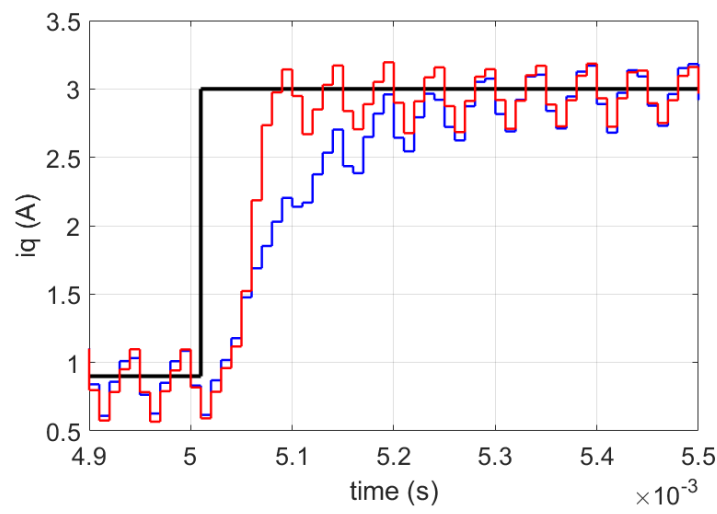

Fig. 16. $i_{q}$ step from 0.9 to $3 \mathrm{~A}$ at $\omega_{m}$ of $100 \frac{\mathrm{rad}}{\mathrm{r}}$. Reference current $i_{q}$ (black line), FPGA DB $i_{q}$ (blue line), OS-DB $i_{q}$ (red line).

controller for pmsm drives," IEEE Transactions on Industrial Electronics, vol. 63, no. 6, pp. 3906-3914, June 2016.

[18] J. S. Lee and R. D. Lorenz, "Robustness analysis of deadbeat-direct torque and flux control for ipmsm drives," IEEE Transactions on Industrial Electronics, vol. 63, no. 5, pp. 2775-2784, May 2016.

[19] Y. A. R. I. Mohamed and E. F. El-Saadany, "Robust high bandwidth discrete-time predictive current control with predictive internal model; a unified approach for voltage-source pwm converters," IEEE Transactions on Power Electronics, vol. 23, no. 1, pp. 126-136, Jan 2008.

[20] S. D. S. Paul C. Krause, Oleg Wasynczuk, in Analysis of electric machinery and drive systems 2nd ed. New York IEEE Press - IEEE Press power engineering series, 2002, pp. 191-206.

[21] T. Soderstrom and P. Stoica, in System Identification. Prentice Hall International Series in Systems and Control Engineering, 1994, pp. 320324

[22] A. Galassini, G. L. Calzo, A. Formentini, C. Gerada, P. Zanchetta, and A. Costabeber, "ucube: Control platform for power electronics," in 2017 IEEE Workshop on Electrical Machines Design, Control and Diagnosis (WEMDCD), April 2017, pp. 216-221 